\title{
Hydrodynamic Device Simulation with New State Variables Specially Chosen for a Block Gummel Iterative Approach
}

\author{
WENCHAO LIANG, DANIEL C. KERR, NEIL GOLDSMAN and ISAAK D. MAYERGOYZ \\ Department of Electrical Engineering, University of Maryland, College Park, MD 20742
}

\begin{abstract}
A new numerical formulation for solving the hydrodynamic model of semiconductor devices is presented. The method is based on using new variables to transform the conventional hydrodynamic equations into forms which facilitate numerical evaluation with a block Gummel approach. To demonstrate the new method, we apply it to model a $0.35 \mu \mathrm{m} 2$-D LDD MOSFET, where robust convergence properties are observed.
\end{abstract}

\section{INTRODUCTION}

We present a new numerical approach for solving the HD equations which is specially tailored for use with a block-Gummel iterative method. With this approach, we decouple the equations into a Poissoncurrent-continuity (PC) block and an energy balance (EB) block, and solve the system by iterating between the blocks. Instead of using standard variables $n, p$, $T_{e}, T_{p}$, we introduce new state variables. When used in conjunction with the block Gummel method, the new variables facilitate transformation of the HD equations into linear forms, which are then solved directly or by using the fixed-point iteration method $[1,2]$. To help resolve rapid variations in the state variables, we have developed a Scharfetter-Gummel (SG) type approach to discretize the linear equations. The SG discretization yields a discrete system with coefficient matrices which are well conditioned, thereby helping to avoid numerical problems associated with their solution. The coordinated use of the new variables, the Gummel iterative approach, and the SG discretization yields a robust approach to solv- ing the HD equations. We demonstrate the new approach by applying it to a $0.35 \mu \mathrm{m}$ 2-D LDD MOSFET, where reliable convergence properties are observed.

\section{NEW STATE VARIABLES}

To facilitate solving the HD model, we define the new state variables $\left\{\phi, u, v, g_{n}, g_{p}\right\}$. The major objectives in choosing these variables are the following:

i. To tailor the HD equations specifically for use with a Gummel iterative method;

ii. To transform the equations so that the left-hand side (LHS) of each equation is linear with respect to a new state variable with which this equation is identified;

iii. To yield well-conditioned coefficient matrices upon discretization of the transformed equations;

iv. To help resolve the fast spatial variation in carrier concentration and carrier temperature which occurs in semiconductor devices. 
Below we present the new state variables; the detailed methodology used to determine them is motivated by transforming the conventional steady state 2-dimensional HD equations [3,4], into forms which are nearly self-adjoint.

The new state variables for the carrier concentration are $u$ and $v$, and they are related to $n$ and $p$ as follows:

$$
\begin{gathered}
n=n_{I} e^{\phi / V_{T}} \frac{T_{L}}{T_{n}} u \\
p=n_{I} e^{-\phi / V_{T}} \frac{T_{L}}{T_{p}} v
\end{gathered}
$$

Where $V_{T}=k_{B} T_{L} / q$ and $n_{l}, \phi, T_{L}, T_{n}$ are intrinsic carrier concentration, electrostatic potential, lattice temperature and electron temperature, respectively.

With this choice of new state variables, the HD current density $J_{n}$ becomes

$$
\mathbf{J}_{n}=q n_{I} \mu_{n} V_{T} e^{\phi / V_{T}} \nabla u+q n_{I} \mu_{n} T_{L}\left(\frac{1}{T_{L}}-\frac{1}{T_{n}}\right) e^{\phi / V_{T}} \nabla \phi u
$$

It is worth noting that when $T_{n}=T_{L}$, the second term in the above current equation will vanish, and eq. (3) will reduce to the expression for current in the DD model.

The new state variables for the carrier temperatures are $g_{n}$ and $g_{p}$, and they are related to $T_{n}$ and $T_{p}$ as follows:

$$
\begin{gathered}
T_{n}=T_{L}\left[e^{\phi / V_{T}} u\right]^{-5 / 4} g_{n} \\
T_{p}=T_{L}\left[e^{-\phi / V_{T}} v\right]^{-5 / 4} g_{p}
\end{gathered}
$$

With these new state variables, the HD expression for energy flux $S_{n}$ becomes:

$$
\begin{aligned}
\mathbf{S}_{n} & =-2 q n_{I} \mu_{n} V_{T}^{2}\left(u e^{\phi / V_{T}}\right)^{-1 / 4} \nabla g_{n} \\
& +\frac{5}{2} q n \mu_{n} V_{T}\left(u e^{\phi / V_{T}}\right)^{-5 / 4} \nabla \phi g_{n}+\frac{\mathbf{J}_{n}}{-q} \frac{1}{2} m_{n}^{*} v_{d n}^{2}
\end{aligned}
$$

The expressions for hole current and hole energy flux can be obtained in an analogous manner.

\section{THE TRANSFORMED HD MODEL}

After substituting the new variables into the conventional HD model, we arrive at a new formulation. When used with a block-Gummel iteration scheme, the equations of the transformed model are linear with respect to newly defined state variables. The complete set of transformed HD equations is given below:

$$
\begin{aligned}
& \nabla^{2} \phi=\frac{q n_{I}}{\varepsilon_{s}}\left(\frac{T_{L}}{T_{n}} e^{\phi / V_{T}} u-\frac{T_{L}}{T_{p}} e^{-\phi / V_{T}} v\right)-\frac{q D}{\varepsilon_{S}} \\
& \nabla \cdot\left[n_{I} \mu_{n} V_{T} e^{\phi / V_{T}} \nabla u\right. \\
& \left.+n_{I} \mu_{n} T_{L}\left(\frac{1}{T_{L}}-\frac{1}{T_{n}}\right) e^{\phi / V_{T}} \nabla \phi u\right]=R(\phi, u, v) \\
& \nabla \cdot\left[n_{I} \mu_{p} V_{T} e^{-\phi / V_{T}} \nabla v\right. \\
& \left.-n_{I} \mu_{p} T_{L}\left(\frac{1}{T_{L}}-\frac{1}{T_{p}}\right) e^{-\phi / V_{T}} \nabla \phi v\right]=R(\phi, u, v) \\
& 2 q n_{I} V_{T}^{2} \nabla \cdot\left[\mu_{n}\left(u e^{\phi / V_{T}}\right)^{-1 / 4} \nabla g_{n}\right. \\
& \left.-\frac{5 n}{4 n_{I}} \frac{\mu_{n}}{V_{T}}\left(u e^{\phi / V_{T}}\right)^{-5 / 4} \nabla \phi g_{n}\right]= \\
& n \frac{\frac{3}{2} k_{B} T_{L} g_{n}\left(u e^{\phi / V_{T}}\right)^{-5 / 4}+\frac{1}{2} m_{n}^{*} v_{d n}^{2}-\omega_{0}}{\tau_{n \omega}} \\
& -\mathbf{J}_{n} \cdot \mathbf{E}-\nabla \cdot\left(\frac{\mathbf{J}_{n}}{q} \frac{1}{2} m_{n}^{*} v_{d n}^{2}\right) \\
& -2 q n_{I} V_{T}^{2} \nabla \cdot\left[\mu_{p}\left(v e^{-\phi / V_{T}}\right)^{-1 / 4} \nabla g_{p}\right. \\
& \left.+\frac{5 p}{4 n_{I}} \frac{\mu_{p}}{V_{T}}\left(v e^{-\phi / V_{T}}\right)^{-5 / 4} \nabla \phi g_{p}\right]= \\
& p \frac{\frac{3}{2} k_{B} T_{L} g_{p}\left(v e^{-\phi / V_{T}}\right)^{-5 / 4}+\frac{1}{2} m_{p}^{*} v_{d p}^{2}-\omega_{0}}{\tau_{p \omega}} \\
& -\mathbf{J}_{p} \cdot \mathbf{E}-\nabla \cdot\left(\frac{\mathbf{J}_{p}}{q} \frac{1}{2} m_{p}^{*} v_{d p}^{2}\right)
\end{aligned}
$$

The advantages of the above equations are best understood in the context of the block-Gummel algorithm used to solve these equations. With this algorithm, which is analogous to the one used in [5], the PC block, which contains the above Poisson and continuity equations, is solved self-consistently for the new state variables $\phi, u, v$, while $T_{n}$ and $T_{p}$, are assumed known. Within this PC block, the LHS of the Poisson 
equation is in linear self-adjoint form. The LHS's of the current-continuity equations are linear in $u$ and $v$, but not in self-adjoint form because of the second term under the divergence operator, which can be called the "spoiler" term. Except for in the MOSFET drain region, the carriers are usually close to equilibrium. Thus, throughout a large portion of the device $T_{n} \approx T_{L}$, hence the spoiler term is small, and eq. (8) has many of the numerical attributes of the selfadjoint form. In the drain region, where the spoiler term can become large, our discretization scheme, which employs exponential fitting, helps prevent potential instabilities. Furthermore, since the PC block is similar to the self-adjoint formulation of the drift diffusion model, it is easily solved by using iterative techniques which are similar to those already developed for the DD model [2]. The iterative technique also facilitates the use of special scaling methods which eliminate potential overflow problems which could arise from the use of the new variables. To further emphasize the attributes of the PC block, we do not transform the electron and hole temperatures into their new variables, but leave them in terms of the original or natural variables $T_{n}$ and $T_{p}$. Leaving the variables $T_{n}$ and $T_{p}$ in eq. (8) also emphasizes that the carrier temperatures are assumed known in the PC block. (An analogous approach is taken in the EB block).

The energy balance equation is treated within the context of the EB block. Within this EB block all variables are assumed known except for the new variables $g_{n}$ and $g_{p}$. The energy balance equations are linear in the new variables $g_{n}$ and $g_{p}$, and they also contain second (spoiler) terms under the divergence operator. It is important to observe that the variable transformation has eliminated the explicit nonlinearity in $T_{n}$ and $T_{p}$ in the energy balance equations. It is also worth noting that since the carrier concentration is inversely proportional to $g_{n}$, state variables $n$ and $g_{n}$ thereby tend to cancel in the spoiler terms (especially upon convergence), and many of the numerical attributes of the self-adjoint form may be realized.

To help account for the rapid variations in carrier concentration and carrier temperatures without having to employ an overly dense mesh, we adapt the SG discretization methodology to our model. To achieve this, we analytically solve the homogeneous parts of the current-continuity and energy balance equations between mesh-points, while accounting for the nonhomogeneous parts on the mesh-points. In addition to resolving rapid variations, the discretization yields coefficient matrices for the current-continuity and energy balance equations which are well conditioned and likely to be diagonally dominant.

\section{METHOD OF SOLUTION AND NUMERICAL RESULTS}

We demonstrate the new approach with the simulation of a realistic $0.35 \mu m n$-channel LDD MOSFET. The device had peak source and drain dopings of $N_{D}=1 \times$ $10^{20} \mathrm{~cm}^{-3}$ and LDD region doping of $N_{D}=1 \times$ $10^{18} \mathrm{~cm}^{-3}$ The substrate doping was $N_{A}=5 \times$ $10^{16} \mathrm{~cm}^{-3}$, and the oxide thickness was $0.025 \mu \mathrm{m}$. The doping profile is shown in Figure 1. We solved the complete HD model for electrons, while for holes $T_{p}$ was assumed constant and equal to lattice temperature.

We discretize the system on a $58 \times 41$ nonuniform rectangular mesh. As described above, we use an inner Gummel block to self-consistently solve the discretized Poisson and continuity equations (PC block) while the carrier temperature is assumed known. Once this inner block converges, we solve the discretized energy balance equation with carrier concentration and potential assumed known (EB block), and then iterate between blocks until convergence is achieved. Convergence criteria was chosen to be when the relative normalized error of $10^{-4}$ was reached.

Figures 2 through 5 show example calculation results for the device biased at the onset of pinchoff with $V_{G}=V_{D}=3.0 \mathrm{~V}$. Figure 2 shows the calculated electrostatic potential plot of the electric field. Figures 3 and 4 show the electron and hole concentrations respectively. Figure 5 shows the very smooth results obtained for electron temperature. It is interesting to observe the double peak resulting from the $n^{+}-n$ and the $n-p$ junction. While we have shown detailed cal- 


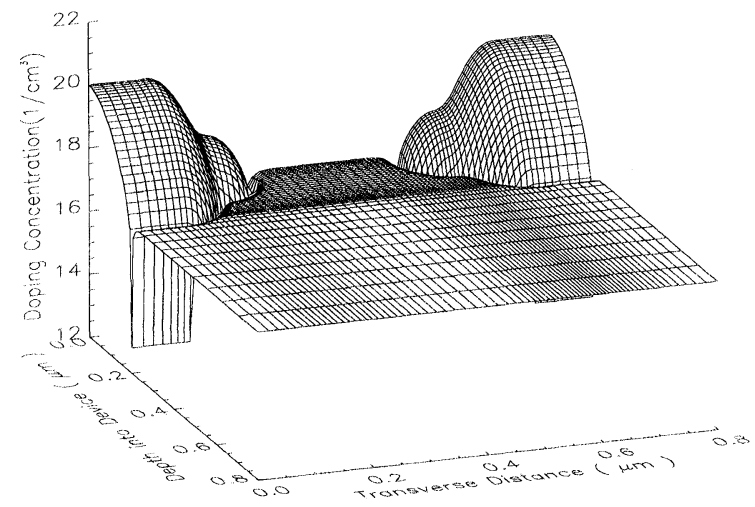

FIGURE 1 Doping profile in a simulated $0.35 \mu \mathrm{m}$ LDD MOSFET device

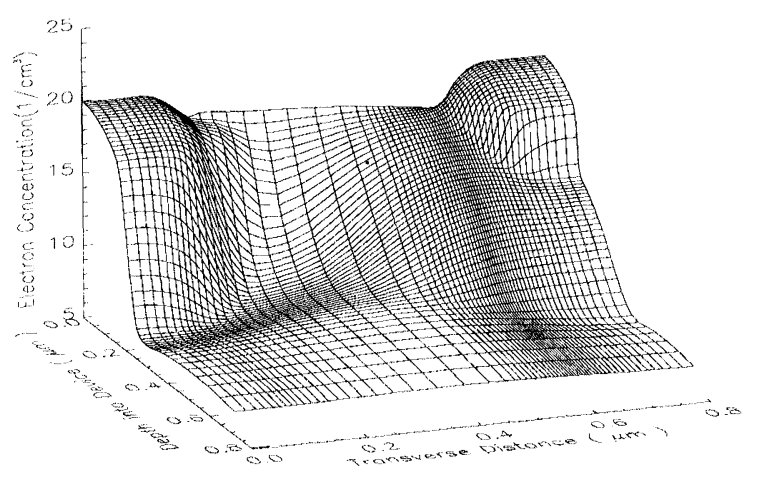

FIGURE 3 Electron concentration in a $0.35 \mu \mathrm{m}$ LDD MOSFET with $V_{g s}=3 \mathrm{~V}, V_{d s}=3 \mathrm{~V}$ calculated by the HD device simulator

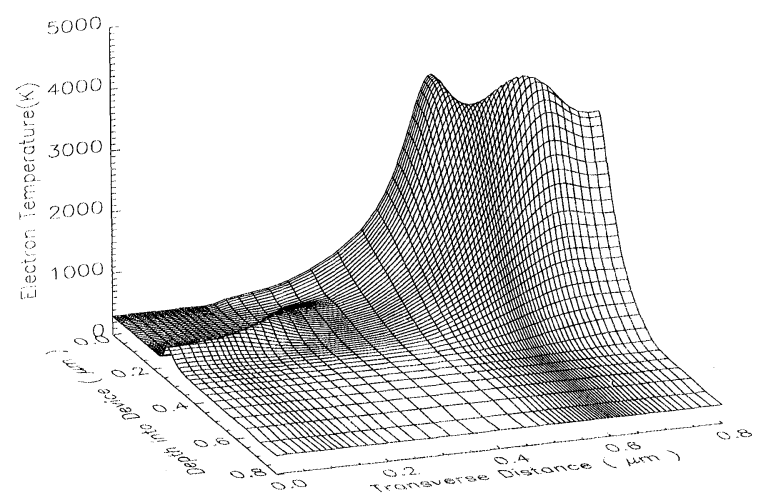

FIGURE 5 Electron Temperature in a $0.35 \mu \mathrm{m}$ LDD MOSFET with $V_{g s}=3 \mathrm{~V}, V_{d s}=3 \mathrm{~V}$ calculated by the HD device simulator

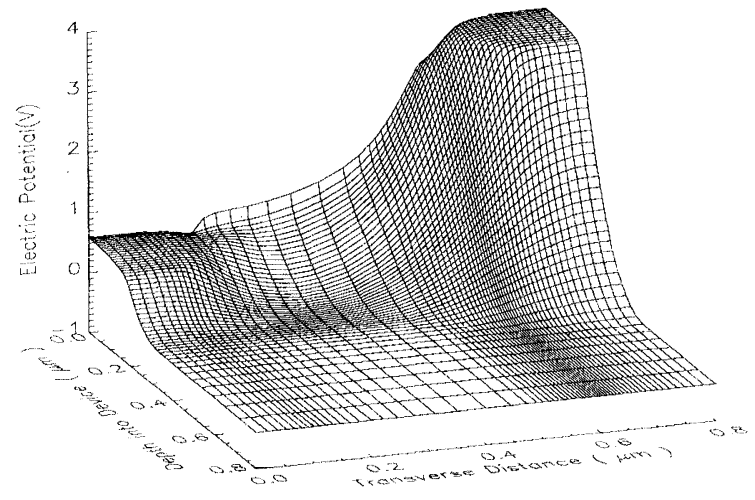

FIGURE 2 Electric Potential in a $0.35 \mu m$ LDD MOSFET with $V_{g s}=3 V, V_{d s}=3 V$ calculated by the HD device simulator

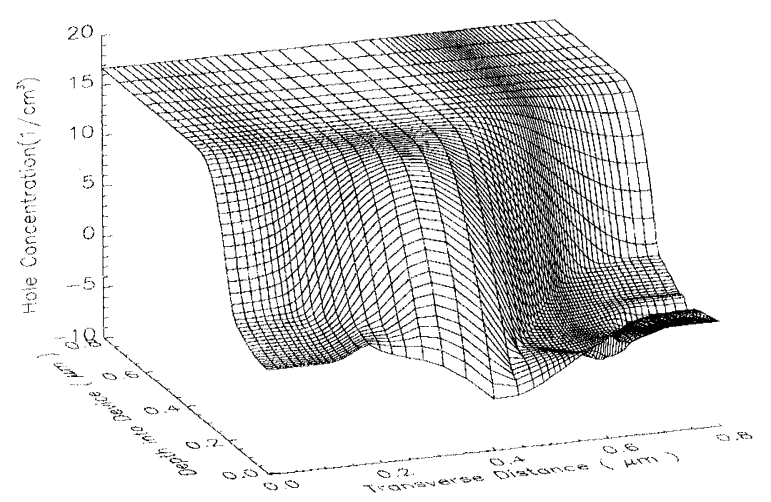

FIGURE 4 Hole concentration in a $0.35 \mu \mathrm{m}$ LDD MOSFET with $V_{g s}=3 \mathrm{~V}, V_{d s}=3 \mathrm{~V}$ calculated by the HD device simulator

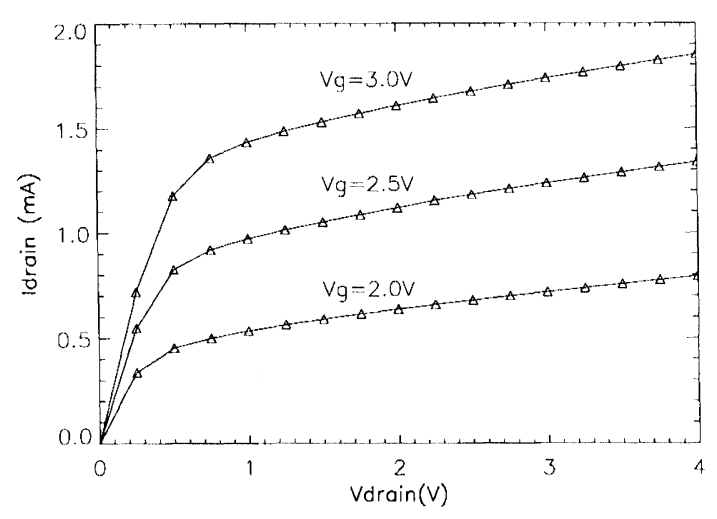

FIGURE $6 \mathrm{I}-\mathrm{V}$ characteristics for a $0.35 \mu \mathrm{m}$ LDD MOSFET calculated by the HD device simulator 
culation results for the bias at the onset of pinchoff, we demonstrate broadrange results with the calculated current-voltage characteristics for the device in Figure 6.

In summary, we have presented a new approach to the hydrodynamic problem. In addition to being computationally robust, it also has the advantages of being relatively simple to code, requiring relatively little memory, and being easily parallelized, thereby making it extendable to large problems, especially 3-D applications.

\section{References}

[1] I. D. Mayergoyz, "Solution of the Nonlinear Poisson Equation of Semiconductor Device Theory," Journal of Applied Physics, vol. 59, no. 1, pp. 195-199, 1986.
[2] C. E. Korman and I. D. Mayergoyz, "A Globally Convergent Algorithm for the Solution of the Steady-State Semiconductor Device," Journal of Applied Physics, vol. 68, no. 3, p. 1324,1990

[3] A. Forghieri, R. Guerrieri, P. Ciampolini, A. Gnudi, M. Rudan, and G. Baccarani, "A New Discretization Strategy of the Semiconductor Equations Comprising Momentum and Energy Balance," IEEE Trans. Computer-Aided Design, vol. 7, pp. 231-242, 1988.

[4] K.-W. Chai, P. Mawby, and A. McCowen, "Hydrodynamic Simulation of Electron Heating in Conventional and LightlyDoped-Drain MOSFETs with Application to Substrate Current Calculation," International Journal of Numerical Modelling: Electronic Networks, Devices and Fields, vol. 5, pp. 53-66, 1992.

[5] Q. Lin, N. Goldsman, and G.-C. Tai, "Highly Stable and Routinely Convergent 2-Dimensional Hydrodynamic Device Simulation," Solid-State Electronics, vol. 37, pp. 359- 371, 1994. 

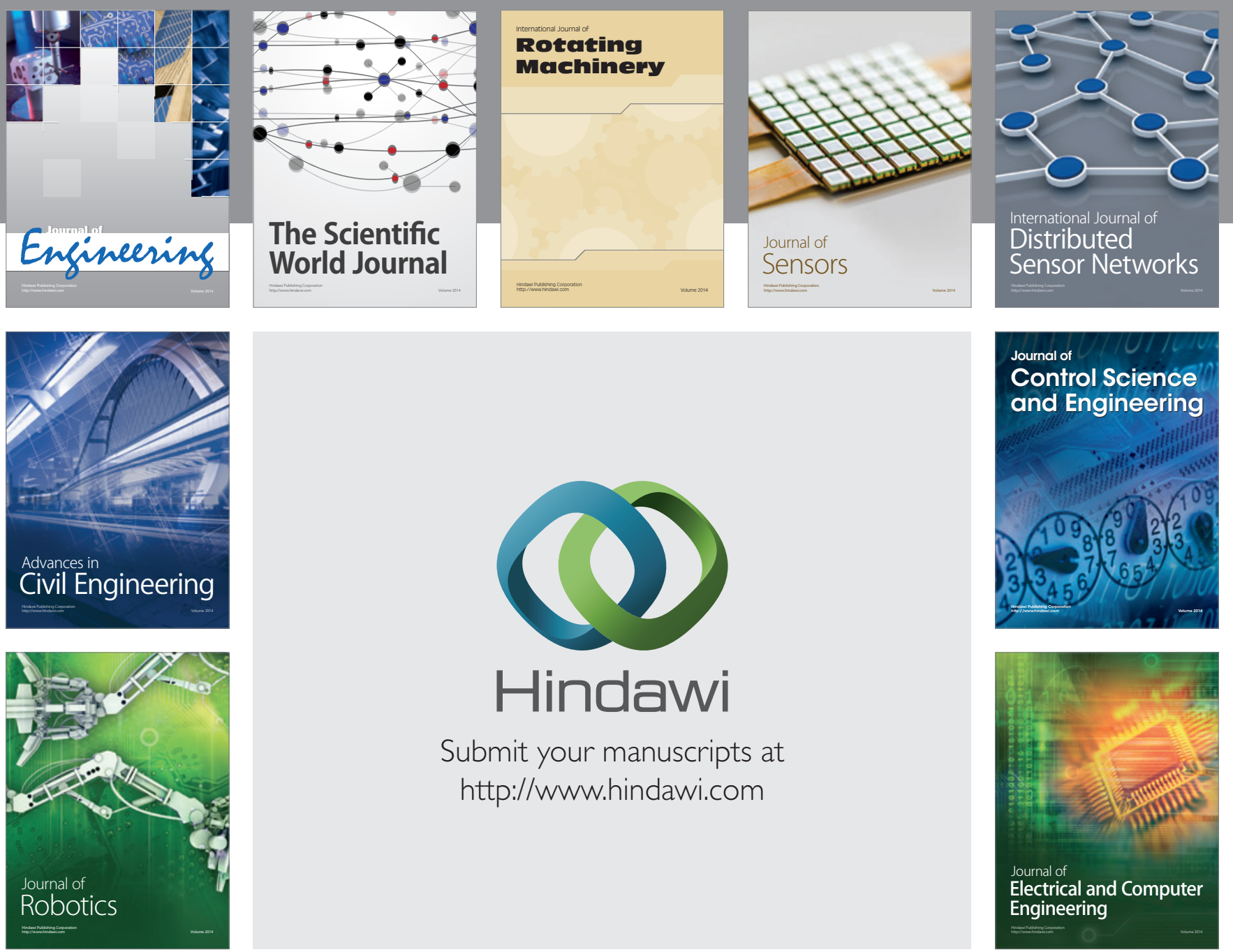

Submit your manuscripts at

http://www.hindawi.com
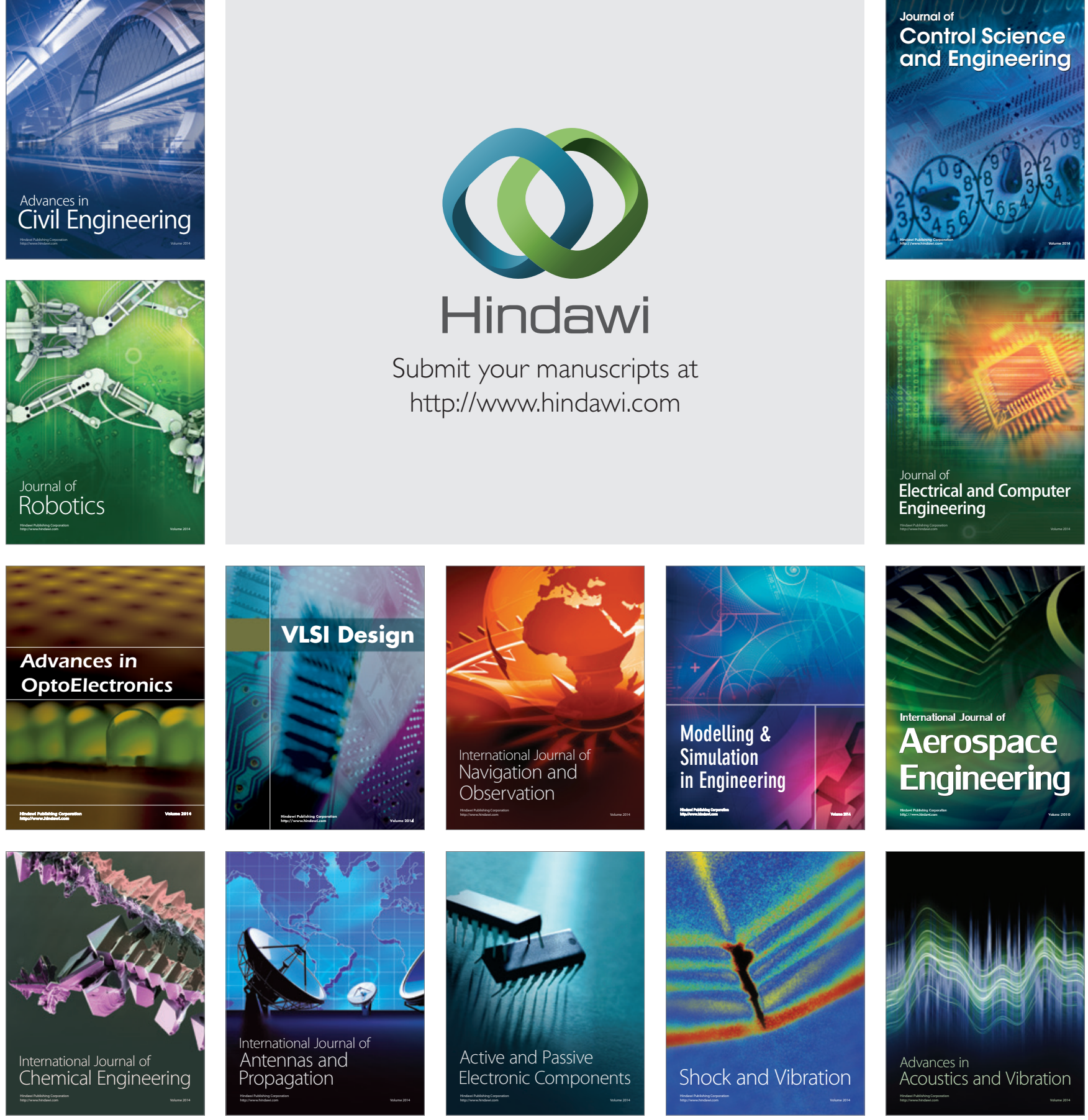